\title{
A silicon carbide photonic platform based on suspended subwavelength waveguides
}

\author{
Francesco Garrisi ${ }^{1,}$, , Ioannis Chatzopoulos ${ }^{2,+}$, Robert Cernansky $2,+$, And Alberto Politi ${ }^{2}$ \\ ${ }^{1}$ Dipartimento di Fisica, Università di Pavia, 27100 Pavia, Italy \\ ${ }^{2}$ School of Physics and Astronomy, University of Southampton, Southampton, S017 1BJ, United Kingdom \\ ${ }^{*}$ Corresponding author: francesco.garrisi01@universitadipavia.it \\ ${ }^{+}$Currently at the National Physical Laboratory, Hampton Road, Teddington TW11 OLW, United Kingdom \\ $\ddagger$ Currently at Centre for Quantum Dynamics, Griffith University, Brisbane QLD 4111, Australia
}

Compiled September 14, 2020

\begin{abstract}
Silicon Carbide (SiC) displays a unique combination of optical and spin-related properties that make it interesting for photonics and quantum technologies. However, guiding light by total internal reflection can be difficult to achieve, especially when $\mathrm{SiC}$ is grown as thin films on higher index substrates, like silicon. Fabricating suspended subwavelength waveguides requires a single lithography step and offers a solution to the confinement problem, while preserving the design flexibility required for a scalable and complete photonic platform. Here we present a design for such platform, which can be used for both classical and quantum optics operation. We simulate basic optical components and analyze how to exploit the high nonlinearities of $\mathrm{SiC}$ and its defects. (๑) 2020 Optical Society of America
\end{abstract}

http://dx.doi.org/10.1364/ao.XX.XXXXXX

\section{INTRODUCTION}

Silicon Carbide ( $\mathrm{SiC})$ is establishing itself as an important material in the field of quantum photonics. Among its many polytypes, $3 \mathrm{C}$ and $4 \mathrm{H}-\mathrm{SiC}$ are hosts of a large variety of point defects emitting in the visible and in the near infrared (NIR) [1-4]; these defects can be used as single photon sources and their spin state can be addressed through radio frequency and optical electromagnetic fields, while the coherence time has been shown to exceed milliseconds [5-7].

Photonic structures can enhance the interaction between these colour centres and light, providing a path for the development of a scalable approach for quantum technologies. Moreover, SiC provides interesting optical properties. Being non-centrosymmetric crystals, both polytypes of $\mathrm{SiC}$ possess a strong static second-order nonlinearity $\left(\chi_{\mathrm{xyz}}^{(2)} \simeq 60 \mathrm{pm} / \mathrm{V}\right.$ for $3 \mathrm{C}-\mathrm{SiC}$ [8] and $\chi_{\mathrm{zzz}}^{(2)} \simeq 32.8 \mathrm{pm} / \mathrm{V}$ for $4 \mathrm{H}-\mathrm{SiC}$ [9]); they do not suffer from two-photon absorption at telecommunication wavelengths due to their large electronic bandgap (around $2.4 \mathrm{eV}$ for $3 \mathrm{C}$ and $2.9 \mathrm{eV}$ for $4 \mathrm{H} \mathrm{[10]);} \mathrm{as} \mathrm{well} \mathrm{as} \mathrm{diamond,} \mathrm{SiC}$ is one of the hardest known materials [11], providing the mechanical stabil- ity required to support complex nanostructures at small scale [12], along with excellent thermal conductivity. SiC is also being investigated as a dielectric photonic platform for plasmonic applications [13]. Finally, $\mathrm{SiC}$ is known to be an established platform for high power microelectronics, making promising the integration of photonic and electronic devices on the same platform.

The fabrication of $\mathrm{SiC}$ for photonic applications, however, can be problematic. For example, few hundred nanometers of 3C-SiC can be grown heteroepitaxially on silicon ( $\mathrm{Si}$ ), but this poses two issues: i) having a higher index of refraction, the substrate prevents the use of total internal reflection (TIR) to obtain light confinement in the vertical direction; ii) due to crystalline mismatch, the interface between 3C-SiC and $\mathrm{Si}$ grows with very low quality, increasing losses of light travelling in such region. These two problems can be addressed at once by adopting wafer-bonding techniques [14]. On the other hand, the homoepitaxial growth of $4 \mathrm{H}-\mathrm{SiC}$ provides high quality films, but obtaining thin membranes is not straightforward. Smartcut process [15] can be applied to obtain $\mathrm{SiC}$ on insulator, but the ion implantation step increases optical losses and produces lattice damages detrimental to color center properties. Wafer bonding and thin down has demonstrated excellent material properties and low losses in photonic crystal cavities [16] and ring resonators [17]. However, the uniformity of the thickness of the $\mathrm{SiC}$ layer over appreciable chip sizes is a limiting factor for the scalability of $\mathrm{SiC}$ photonics.

An alternative approach is to suspend membranes in air, either removing part of the Si substrate, or by electrochemical etching of doped $\mathrm{SiC}$. This approach has been used to produce photonic crystal cavities $[18,19]$ as well as optical waveguides using a two-step lithography technique [20]. In this case, the first exposure defines the lateral confinement of the waveguides, while the second one opens holes to access the substrate that has to be removed.

Here we propose subwavelength geometries that allow the use of a single lithography step to define the waveguide geometry and access the substrate, simplifying the fabrication, as it has already been demonstrated for other platforms [21-24]. Subwavelength structures can be defined as periodic dielectric structures whose periodicity is much smaller than the wave- 
length of light; more rigorously, they are periodic structures where the energy of the photonic bandgap lies above the energy of the photons propagating in the medium. As such, they behave as an effective homogeneous medium (EHM) and they prevent the scattering of light [25]. Subwavelength structures can be used easily to obtain a complete photonic platform in $\mathrm{SiC}$, capable not only to guide light, but also to realize ring resonators, grating couplers and slow-light waveguides.

In Section 2 the design of the most basic component of the platform, a straight subwavelength suspended waveguide, is presented, followed by a discussion on the results of the numerical simulations which led to the choice of the dimensions of the waveguide. Then, we consider the amount of losses expected from the waveguide design, and we give an estimate of the nonlinear waveguide parameter. In Section 3 we assess the tolerance of the design to fabrication imperfections, in terms of the variation of the modal refractive index resulting from variation in the geometry of the waveguide. Section 4 briefly introduces the analysis of additional photonic components and presents detailed results of numerical simulations used to design a uniform grating coupler. Section 5 describes how the platform can be adapted easily to reach a slow-light regime by changing the periodicity of the lateral suspending structures. Section 6 discusses the performances of a proposed design for an electro-optical modulator integrated alongside the suspended waveguides. Finally, in Section 7 we give the conclusions and perspectives.

\section{SUBWAVELENGTH WAVEGUIDE}

When a dielectric medium is periodic in one direction, the light travelling inside it can be described in terms of the photonic band structure [26]. The periodicity will produce the emergence of the photonic bandgap, a range of frequencies at which light cannot propagate in the medium. If the energy of the light is lower than the photonic bandgap, radiation can propagate, ideally without scattering, and the periodic medium acts as an EHM [25].

In Figure 1-a) we show the design of a $\mathrm{SiC}$ waveguide that exploits this principle to guide light at $1550 \mathrm{~nm}$ wavelength. The design is based on previous works realized in silicon on insulator (SOI) [22, 23]. Light is confined in the vertical direction by TIR. The lateral arms serve two functions: to mechanically suspend the waveguide and to introduce the periodic perturbation. The perturbation has a periodicity that is much smaller than the wavelength of light, hence, similarly to a multilayer, the arms act as a homogeneous medium with index of refraction $n_{e}$ intermediate between the one of $\mathrm{SiC}$ and air. Thus, for the case of the straight waveguide presented here, the structure is akin to the one shown in Figure 1-b), where the yellow region highlights the EHM; in practice, this confines light by TIR on the horizontal direction, as well.

The bulk effective index $n_{e}$ of the subwavelength region can be tuned changing the filling factor (FF) of the arms $f_{\mathrm{wg}}$ in the periodic cell, and can be estimated by calculating the effective index of the light travelling normal to a SiC-air multilayer with the same periodicity and FF of the lateral arms [27]. The minimum feature size given by the fabrication process sets the constraints for $f_{\mathrm{wg}}$ and hence to $n_{e}$. For our $\mathrm{SiC}$ structure we believe the higher limit on $f_{\mathrm{wg}}$ will be set by the resolution of the lithographic process, while the lower limit will be determined by the mechanical strength of the material. For instance, other struc- tures in SOI $[22,23]$ were fabricated with a minimum dimension of the arms equal to $100 \mathrm{~nm}$. Since $\mathrm{SiC}$ is a very hard material [11], it is reasonable to assume that the minimum dimension of the arms could be smaller than this value, but a more detailed analysis is required that takes into account not only the mechanical stability of the material but also the inner stress.

\begin{tabular}{cc}
\hline Dimension & Length $[\mathrm{nm}]$ \\
\hline Film thickness $(h)$ & 300 \\
Waveguide width $(w)$ & 650 \\
Periodicity $\left(a_{0}\right)$ & 300 \\
Arm width $\left(u=f_{\mathrm{wg}} a_{0}\right)$ & 150 \\
Arm length $(v)$ & 2000 \\
\hline
\end{tabular}

Table 1. Proposed dimensions for a single TE-TM subwavelength waveguide. We assume a value of 2.6 for the index of refraction of $\mathrm{SiC}$, suspension in air and vertical walls.

The goal of the design process is to obtain a structure sustaining a single TE mode, while maximizing its confinement.. The dimensions of the proposed structure are listed in Table 1. We assumed a value of 2.6 for the refractive index of the $\mathrm{SiC}$ layer since it is close to the refractive indexes of both $3 \mathrm{C}$ and $4 \mathrm{H}-\mathrm{SiC}$ [10]. Then, the layer thickness $(h)$ of $300 \mathrm{~nm}$ was chosen in order to have the fundamental slab mode close to the cut-off condition. The periodicity of the structure $\left(a_{0}\right)$ is set by the subwavelength condition: the continuous lines of Figure 2 are the band structure of our subwavelength waveguides calculated using MIT Photonic Bands (MPB) [28]; choosing a periodicity of $300 \mathrm{~nm}$ puts the TE bandgap well above the energy of $1550 \mathrm{~nm}$ radiation, ensuring the suppression of scattered light and the validity of the EHM approximation.

We have chosen $f_{\mathrm{wg}}=u / a_{0}$ equal to 0.5 , so that the lateral arms are $150 \mathrm{~nm}$ long $(u)$ in the propagation direction. As explained previously, this corresponds to having $n_{e}$ equal to 2.144 , a good compromise between a higher lateral confinement and a high mechanical strength. The proposed waveguide's width (w) of $650 \mathrm{~nm}$ is the one that maximizes the confinement of the fundamental mode while maintaining the structure singleTE-moded. In fact, the structure sustains a single TM mode $\left(\mathrm{TM}_{00}\right)$ and two TE modes $\left(\mathrm{TE}_{00}\right.$ and $\left.\mathrm{TE}_{01}\right)$; the $\mathrm{TE}_{01}$ mode is very loosely bound and is likely to experience very high losses compared to the $\mathrm{TE}_{00}$ mode, since it would be easily coupled to radiative modes. The effective mode area of the $\mathrm{TE}_{00}$ mode is $0.292 \mu \mathrm{m}^{2}$.

The mode profiles have been simulated both with the MPB simulation suite and with a numerical eigensolver (Lumerical MODE). In Figures 1-c), 1-d) and 1-e) we show the profiles of the TE and TM modes calculated with the eigensolver, under the EHM approximation for the lateral arms, (that is, simulating a cross-section of the structure shown in Figure 1-b)). The fundamental mode effective area is $0.348 \mu \mathrm{m}^{2}$. These modes agree very well with the ones obtained from MPB (not shown). Moreover, although not perfectly, the dispersion of the three modes calculated from the eigensolver (the dots in Figure 2) is in agreement with the band structure calculated by MPB, apart from a relative shift of the effective refractive index; at $1550 \mathrm{~nm}$ the effective index of the fundamental TE mode given by the eigensolver $\left(n_{\mathrm{TE}}=1.967\right)$ is slightly higher than the one obtained from the MPB band structure (1.907). 

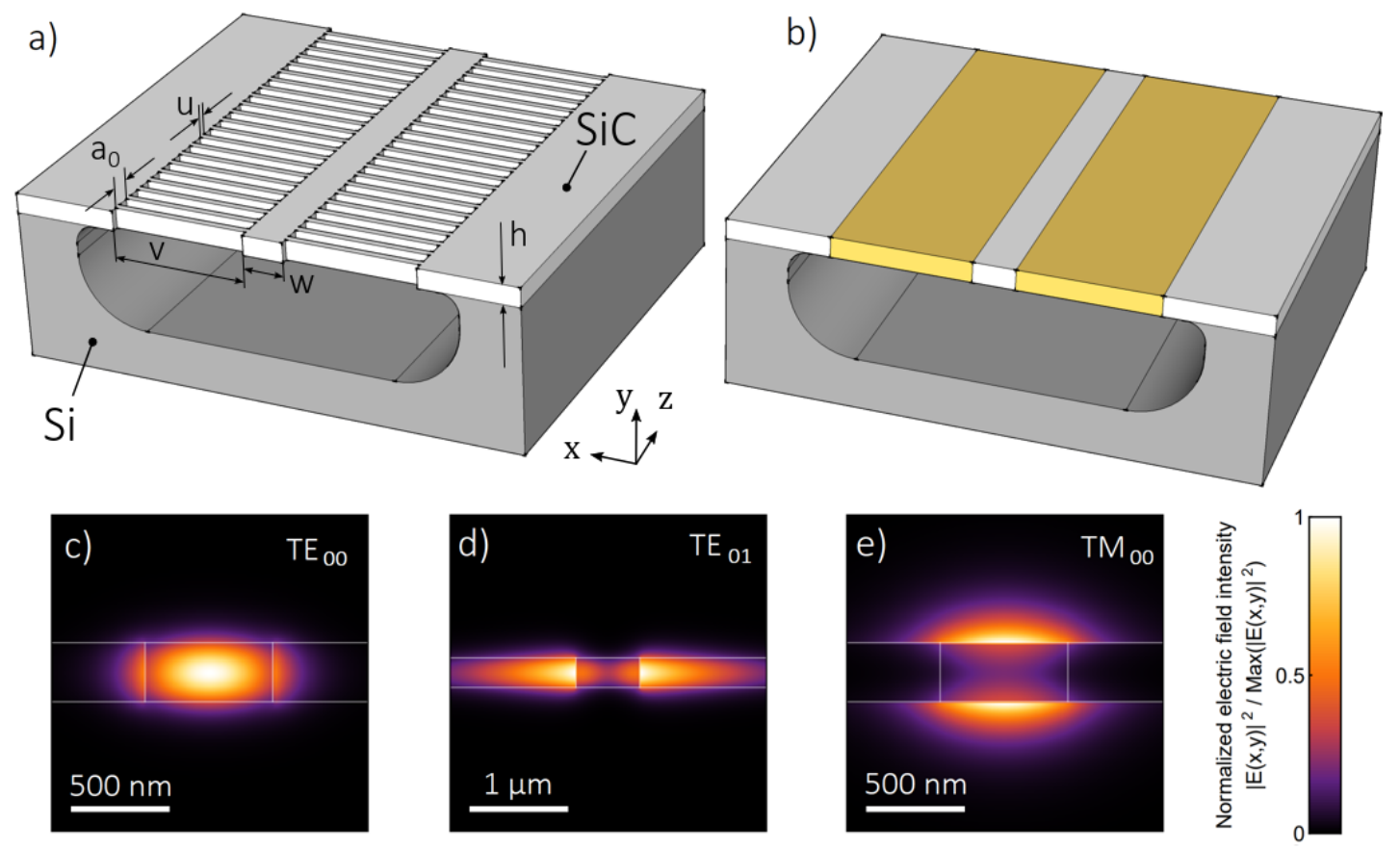

Fig. 1. a) Scheme of the proposed design of a $\mathrm{SiC}$ suspended waveguide. The $\mathrm{SiC}$ film is $300 \mathrm{~nm}$ thick and sits on top of a Silicon substrate. The central branch (650 nm wide) is supported by the lateral arms, which have a longitudinal periodicity of $300 \mathrm{~nm}$ and dimensions of $2 \mu \mathrm{m} \times 150 \mathrm{~nm}$. b) The arms act as an effective uniform medium (yellow) and contribute to guide light by total internal reflection. c-e) Electric field intensity profiles of the $\mathrm{TE}_{00}, \mathrm{TE}_{01}$ and $\mathrm{TM}_{00}$ modes.

In order to estimate the lateral confinement we calculated the effective light-lines in the horizontal direction for the TE and TM modes, which are reported as the dashed lines of Figure 2. The two lines are calculated from an effective index approach: the lateral confinement of the waveguide-arms system has been modeled by an infinite symmetric slab parallel to the $y-z$ plane, surrounded by a cladding material, and whose thickness equals the waveguide's width $(650 \mathrm{~nm})$. The effective light-lines of the original system are then equal to the light-lines of this new slab. The refractive index of the cladding $n_{\mathrm{TE}}(\omega)\left(n_{\mathrm{TM}}(\omega)\right)$ is the only one of importance to determine the light-line, and it is set equal to the effective index of the fundamental TE (TM) slab mode of the original $300 \mathrm{~nm}$ thick layer made of the EHM. Then, the effective light-lines are described in term of the cladding index by the equations

$$
k_{\mathrm{TE}}(\omega)=\frac{\omega}{c} n_{\mathrm{TE}}(\omega), \quad k_{\mathrm{TM}}(\omega)=\frac{\omega}{c} n_{\mathrm{TM}}(\omega)
$$

As seen in Figure 2, the dispersion of the $\mathrm{TE}_{01}$ mode obtained from Lumerical lies very close to the effective TE light-line, thus further confirming that the mode is only loosely bound. Finally, tridimensional FDTD simulations confirm that indeed the eigensolver modes are guided without scattering by the full subwavelength waveguide.

We now consider sources of losses in the subwavelength waveguide other than the intrinsic material losses, since these may depend strongly on the fabrication process and are independent on the platform design. While the fundamental TE mode is found well below the light-line, one expected source of losses is given by the coupling between the fundamental waveguide mode and the modes confined in the remaining SiC layer, past the suspending arms. Indeed, these losses vanish completely only if the width of the lateral arms $v$ is infinitely large. However, we find that a $2 \mu \mathrm{m}$ width of the arms is sufficient to suppress at negligible levels the outcoupling of the guided TE-mode. This width should also ensure the necessary mechanical strength to suspend the waveguide, since similar structures in silicon [21] have been successfully demonstrated. The mode confinement is verified using the same effective-index approach used to calculate the lateral effective light-lines, by adding two additional layers beyond the cladding, which is now $2 \mu \mathrm{m}$ thick in the $x$ direction to both the sides of the central slab. The losses resulting from eigenmode simulations with perfectly matching layer boundary conditions are found lower than $10^{-4} \mathrm{~dB} / \mathrm{cm}$ for $v=2 \mu \mathrm{m}$.

Given the above results, assuming a lossless material, we expect that the main limitations of this kind of structure are given by surface roughness and disorder. Surface roughness couples light from the guided modes to radiative modes $[29,30]$. With respect to traditional ridge waveguides, we expect this effect to be slightly higher due to the presence of the additional material interfaces corresponding to the arms. Still, if needed, the effect of roughness can be counteracted by reducing the presence of light at the interfaces, for instance by increasing the width of the central branch $(w)$, which increases confinement at the expense of the introduction of additional guided modes. Disorder in the periodicity or in the position of the lateral arms also increases losses and has to be kept to low enough values. As shown in ref. [31], where these effects are studied on a similar structure to the one considered here, the jitter in the position and dimension of periodic structures should not exceed $5 \mathrm{~nm}$ to keep losses to a reasonable level. Choosing a working point far below the bandgap can decrease the effect of disorder.

We now consider the nonlinear optical properties of the sys- 


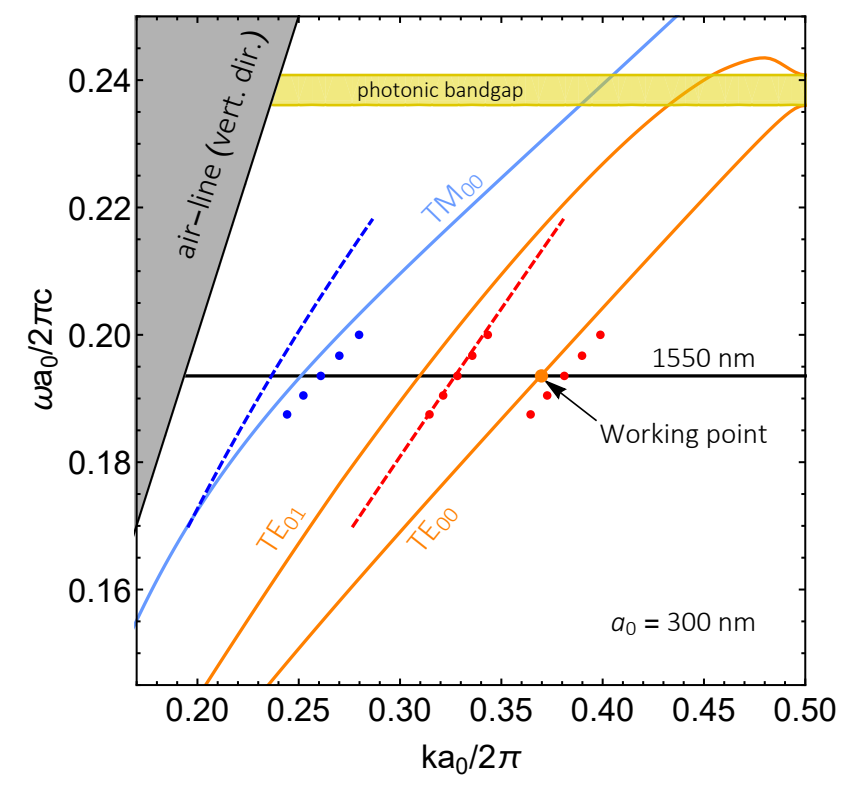

Fig. 2. Simulated band structure of the proposed suspended waveguide along the propagation direction. Assuming $a_{0}$ $=300 \mathrm{~nm}$, the horizontal black line corresponds to $1550 \mathrm{~nm}$. Orange and light blue lines correspond to guided TE and TM modes respectively, calculated with the MIT MPB simulation suite [28]; red and blue dots are the same modes calculated with a numerical eigensolver; dashed red and blue lines are effective horizontal TE and TM light-lines calculated from an effective index approach.

tem. In particular, we estimate the nonlinear waveguide parameter $\gamma$ for the nominal waveguide design. For uniform waveguides, $\gamma$ can be defined in terms of the nonlinear Kerr index $n_{2}$ and of the Poynting vector $\boldsymbol{P}$ [32], according to

$$
\gamma=\frac{k_{0} \int_{\Sigma} n_{2} P_{z}(x, y)^{2} d x d y}{\left|\int P_{z}(x, y) d x d y\right|^{2}}
$$

where $k_{0}$ is the vacuum wavevector, $P_{z}$ is the component of $\boldsymbol{P}$ normal to the integration surface and where the top integral is performed on the cross-section of the waveguide $\Sigma$, that is, where $n_{2}$ is non-vanishing. Since in our case the field changes along the propagation, we average the nonlinear waveguide parameter along a single periodic cell, following the approach described in ref. [33]:

$$
\begin{aligned}
\gamma=\langle\gamma(z)\rangle & =\frac{1}{a_{0}} \int_{z}^{z+a_{0}} \gamma(z) d z= \\
& =\frac{k_{0}}{a_{0}} \int_{z}^{z+a_{0}} \frac{\int n_{2}(x, y, z) P_{z}(x, y, z)^{2} d x d y}{\left|\int P_{z}(x, y, z) d x d y\right|^{2}} d z
\end{aligned}
$$

where $n_{2}(x, y, z)$ is assumed equal to $5.31 \cdot 10^{-19} \mathrm{~m}^{2} / \mathrm{W}$ [20] where $(x, y, z)$ is found within the $\mathrm{SiC}$ structure and zero otherwise. Using the Poynting field calculated from MPB, we find $\gamma=7.346 \mathrm{~W}^{-1} \mathrm{~m}^{-1}$. The nonlinear waveguide parameter is calculated also using Lumerical and the EHM approximation, obtaining $\gamma=6.182 \mathrm{~W}^{-1} \mathrm{~m}^{-1}$; in this case, the presence of the lateral arms is taken into account by assuming that the nonlinear index of the homogeneous medium is the average of the ones of $\mathrm{SiC}$ and air (i.e. equal to $n_{2} / 2=2.655 \cdot 10^{-19} \mathrm{~m}^{2} / \mathrm{W}$ ). By comparison, in ref. [20] the nonlinear waveguide parameter of
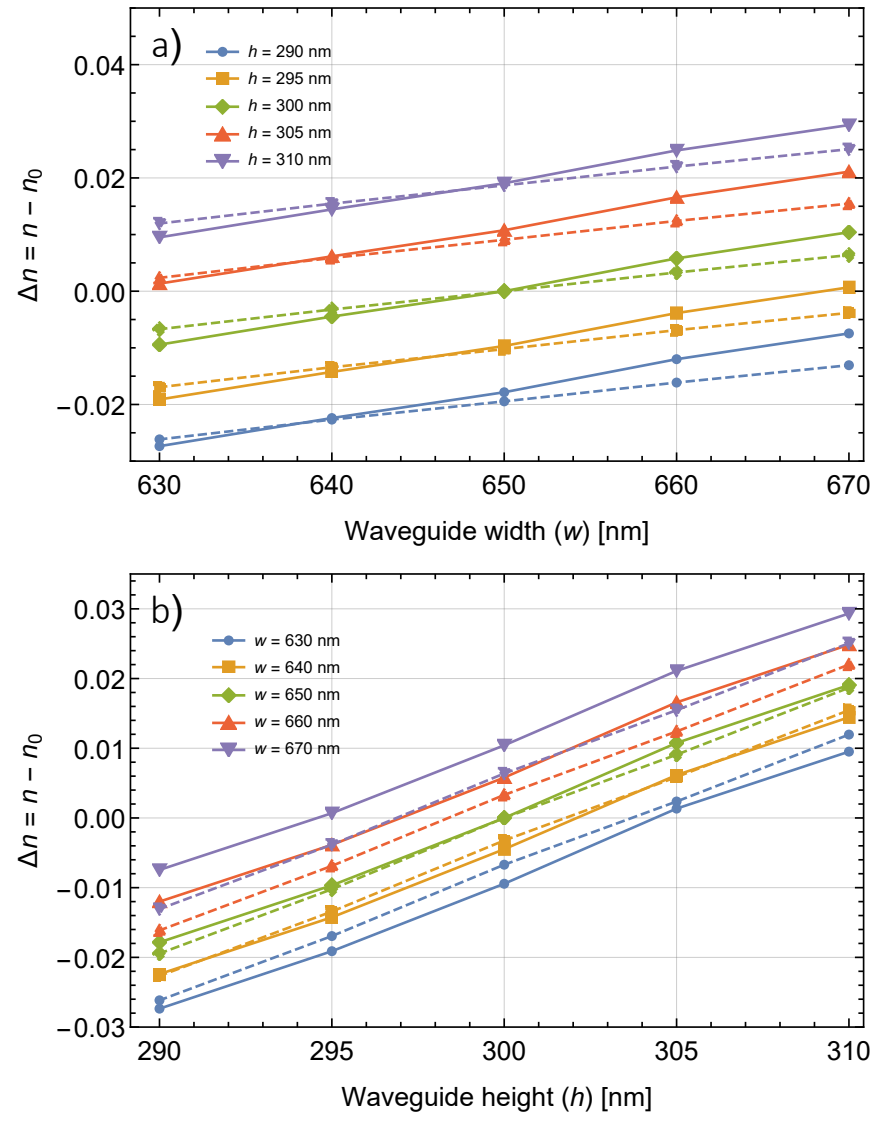

Fig. 3. Simulations of the effective index $n_{\mathrm{TE}}$ of the waveguide's fundamental $\mathrm{TE}_{00}$ mode as a function of the crosssection of the central waveguide. a) Variation of $n_{\mathrm{TE}}$ in terms of the waveguide width (w). b) Variation of $n_{\mathrm{TE}}$ in terms of the waveguide height $(h)$. Continuous lines: MPB - Dashed lines: Lumerical.

slightly lesser confining $\mathrm{SiC}$ waveguides was measured to be $\gamma=3.86 \pm 0.03 \mathrm{~W}^{-1} \mathrm{~m}^{-1}$, while the one of typical silicon nitride waveguides is close to $2 \mathrm{~W}^{-1} \mathrm{~m}^{-1}$ [34].

\section{TOLERANCE}

In order to determine the tolerance of the design to fabrication, we simulate the subwavelength waveguide varying its geometry; in particular, we consider variations in the crosssection and in the filling factor of the lateral arms, and we monitored the change in the effective index of the fundamental TE mode, $\Delta n=n-n_{0}$, where $n_{0}$ is the effective index of the nominal waveguide. The simulations are performed using both the eigensolver and the MPB software suite, and the results for the cross-section variation are reported in Figure 3. We find that the results obtained under the EHM approximation (dashed lines) are in good agreement with the ones obtained from MPB (continuous lines). From the linear fit of the data obtained from MPB, we find that the sensitivities of the waveguide effective index on the width and height are respectively $\sigma_{w}=\Delta n / \Delta w=5.00 \cdot 10^{-4} \mathrm{~nm}^{-1}$ and $\sigma_{h}=\Delta n / \Delta h=1.88 \cdot 10^{-3} \mathrm{~nm}^{-1}$, while from Lumerical we find $\sigma_{w}=3.27 \cdot 10^{-4} \mathrm{~nm}^{-1}$ and $\sigma_{h}=1.91 \cdot 10^{-3} \mathrm{~nm}^{-1}$. Similarly, we perform simulations varying the filling factor of the lateral arms, obtaining $\sigma_{u}=\Delta n / \Delta u=9.69 \cdot 10^{-4} \mathrm{~nm}^{-1}$ with MPB and 
$\sigma_{u}=1.25 \cdot 10^{-3} \mathrm{~nm}^{-1}$ with Lumerical.

The small overall variation of the waveguide's effective index in both cases demonstrates that the subwavelength waveguide design is very tolerant to variations of its geometrical parameters. Moreover, these results justify the use of the homogeneous medium approximation to simplify the design procedure of other photonic devices in this platform, once the difference in the effective index given by the two simulation methods is taken into account.

\section{PHOTONIC COMPONENTS}

A whole range of additional structures can be easily realized under the EHM approximation for the lateral arms region. We can apply standard photonic design and simulation tools to obtain, for example, tapers and bends. Bending losses are obtained from 2-D eigensolver simulations under the EHM approximation, finding a value of about $0.2 \mathrm{~dB} / \mathrm{cm}$ for $20 \mu \mathrm{m}$ bending radius. In order to avoid losses introduced by periodicity mismatch, the arms in bent sections have to maintain their mutual spacing as close as possible to the nominal value of the straight waveguide. We also simulate simple adiabatic tapers of the waveguide, finding that a lenght of $100 \mu \mathrm{m}$ is sufficient to decrease the waveguide width from $12 \mu \mathrm{m}$ to $650 \mathrm{~nm}$ with $96 \%$ transmission. Again, simulations of the structures with FDTD methods confirm the expected behaviour of the devices.

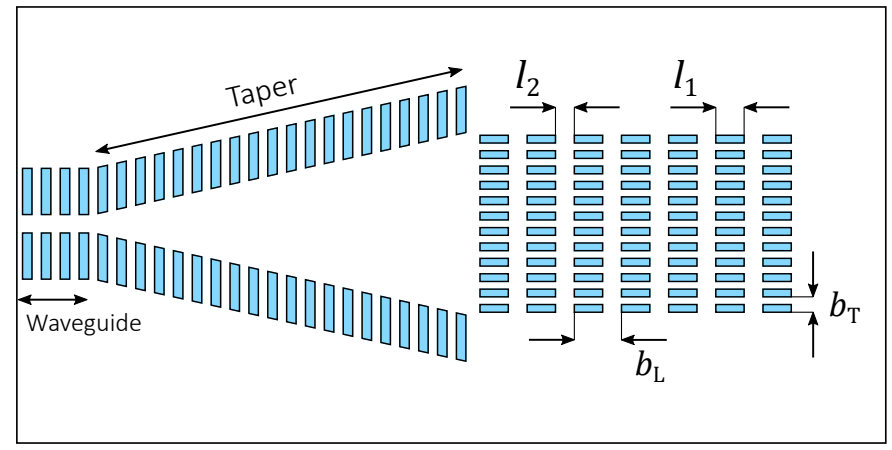

Fig. 4. Schematic representation of the grating coupler geometry (top view, not to scale): blue regions represent holes to be etched in the SiC film.

Efficient coupling of light into the suspended waveguide can be achieved using grating couplers. On this matter, different designs that exploit subwavelength structures have been proposed [24, 35-39]. Briefly, the subwavelength arms, in this case, are used to define the effective index for the subwavelength grooves of the grating coupler and thus are oriented along the propagation direction, as shown schematically in Figure 4. Table 2 lists the dimensions for a uniform grating coupler with simulated $-3.8 \mathrm{~dB}$ maximum coupling efficiency, designed for TE radiation incoming at an $8^{\circ}$ angle to normal incidence.

For the subwavelength grooves we choose a (transversal) periodicity $b_{T}$ and filling factor $f_{\text {grat,T }}$ of $300 \mathrm{~nm}$ and 0.5 , respectively. The final design is obtained by first estimating the effective indices of the high and low index regions of the grating, and then by performing 3D FDTD optimization, following the design method described in ref. [35]. The index estimates are used to initialize the FDTD optimization. The effective index of the groove, $n_{e}^{\prime}$, is estimated with numerical simulations of the fundamental slab mode sustained by the groove cross-section: using Lumerical and exploting periodic boundary conditions in the transverse direction we simulate a single cell of the groove, obtaining $n_{e}^{\prime}=1.110$ for $f_{\text {grat }, \mathrm{T}}=0.5$. Since $n_{e}^{\prime}$ is very close to 1 , the subwavelength grooves could be replaced entirely with air, resulting in a simpler design with very similar dimensions, however suspending large sections of $\mathrm{SiC}$ may result in the deformation of the suspended geometries due to stress release. The cross-section of the high index region is simply a SiC slab suspended in air, thus the associated effective index is the one of its fundamental TE mode, equal to $n_{e}^{\prime \prime}=2.134$.

\begin{aligned} & \hline Film thickness $300 \mathrm{~nm} \\ &$\hline Longit. period $\left(b_{L}\right) 1230 \mathrm{~nm}^{*} \\ &$ Longit. FF $\left(f_{\text {grat }, \mathrm{L}}\right) 32.4 \%$ * \\ & Number of periods 13 \\ & \hline Transv. period $\left(b_{T}\right) 300 \mathrm{~nm} \\ &$ Transv. FF $\left(f_{\text {grat }, \mathrm{T}}\right) 50 \% \\ &$ Transv. length $12 \mu \mathrm{m} \\ &$\hline Max. transmission: $41.8 \%(-3.8 \mathrm{~dB}) \\ & 1$ dB bandwidth: $75 \mathrm{~nm} \\ &$\hline\end{aligned}

Table 2. Proposed dimensions and properties for a TE SiC subwavelength grating coupler operating around $1550 \mathrm{~nm}$. The index of $\mathrm{SiC}$ is assumed to be 2.6. The values marked with * are obtained by numerical optimization.

The estimates for the longitudinal parameters of the design follow from the simplest analytic description of the uniform grating coupler:

$$
l_{1}=\frac{\lambda_{0}}{2\left(n_{1}-n_{c} \sin \alpha\right)}, \quad l_{2}=\frac{\lambda_{0}}{2\left(n_{2}-n_{c} \sin \alpha\right)},
$$

where $l_{1}$ and $l_{2}$ are the longitudinal dimensions of the low- and high-index sections of the grating (so that $b_{L}=l_{1}+l_{2}$ is the grating period and $f_{\text {grat }, \mathrm{L}}=l_{2} /\left(l_{1}+l_{2}\right)$ is the grating longitudinal filling factor), $\lambda_{0}$ is the vacuum wavelength of light, $\alpha$ is the angle to normal incidence, $n_{1}$ and $n_{2}$ are the low and high effective indexes of the light travelling in the grating, and $n_{c}$ is the index of the surrounding material. In our case $n_{1}$ and $n_{2}$ are identified with $n_{e}^{\prime}$ and $n_{e}^{\prime \prime}$ and thus are equal to 1.110 and 2.134 while $n_{c}$ is the index of air (1.0). This gives $b_{L}=1187 \mathrm{~nm}$ and $l_{2} /\left(l_{1}+l_{2}\right)=f_{\text {grat } \mathrm{L}}=32.7 \%$.

Finally, the values marked with * in Table 2 are obtained by numerical 3D FDTD optimization (having maximum transmission at $1550 \mathrm{~nm}$ as target and exploiting periodic boundary conditions in the transverse direction) and they are close to the values obtained above. Figure 5 shows the transmission of the grating as a function of the wavelength, obtained from 3D FDTD simulations; The $1 \mathrm{~dB}$ bandwidth is $75 \mathrm{~nm}$ large, ranging between $1511 \mathrm{~nm}$ and $1586 \mathrm{~nm}$. At the expense of the bandwidth, apodised designs can be employed to increase the maximum coupling efficiency.

\section{SLOW-LIGHT}

In one-dimensional periodic structures, the modal dispersion near the photonic bandgap becomes flatter, corresponding to a lower group velocity of light (so called "slow-light" regime [40]). 


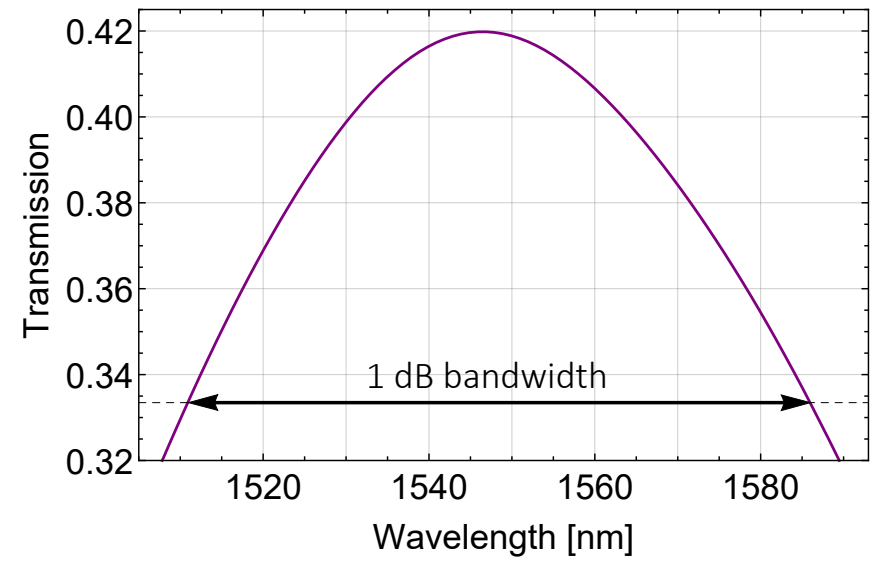

Fig. 5. Simulated transmission of the proposed subwavelength grating coupler.

Exploiting slow-light increases the interaction between radiation and matter. For instance, the fraction of the photons emitted to a guided mode by a dipole localized in a waveguide (also known as $\beta$ factor) is inversely proportional to the group index and can reach values very close to unity $[41,42]$. In our platform, the slow-light regime can be reached naturally by increasing the periodicity of the subwavelength waveguide to move down the bandgap close to the working frequency. As it is shown in Fig. 6 , the group index is more than doubled when the waveguide periodicity approaches $390 \mathrm{~nm}\left(f_{\mathrm{wg}}\right.$ still equal to 0.5$)$.

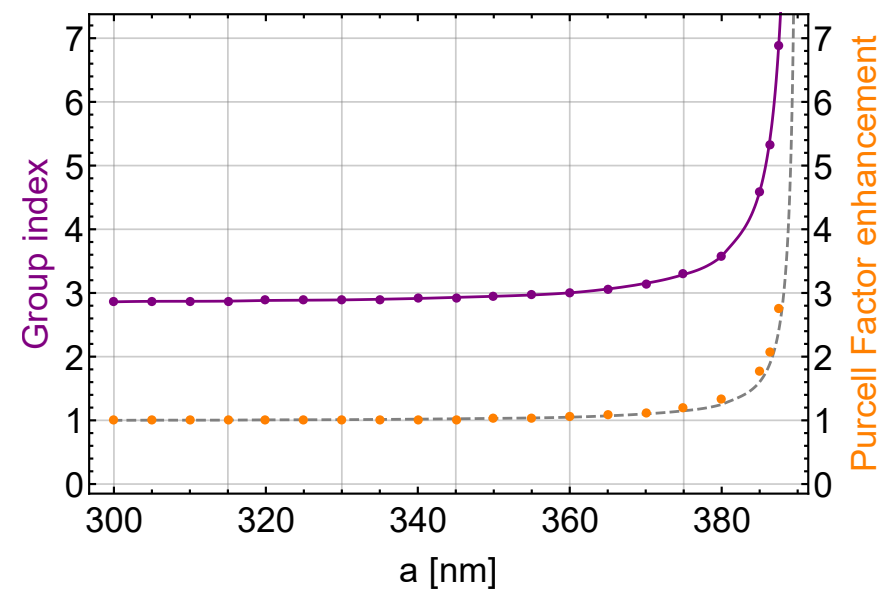

Fig. 6. Group index and Purcell Factor enhancement as a function of the periodicity of the waveguide, obtained from MPB simulations. The dashed gray line is the enhancement of the group index alone, highlighting the main contribution to the Purcell Factor enhancement.

Following the approach of [41], we define an effective mode volume $V_{\text {eff }}$ and Purcell Factor (PF) associated to the light travelling in the waveguide.

$$
\mathrm{PF}=\frac{3 \pi c^{3} a}{V_{\mathrm{eff}} \omega_{0} \epsilon^{3 / 2} v_{g}}
$$

where $\omega_{0}=2 \pi c / \lambda_{0}$ is the frequency of light at the working point, $\epsilon^{1 / 2}=n_{\mathrm{SiC}}$ is the refractive index of $\mathrm{SiC}$ and $v_{g}$ is the group velocity of light; the effective volume $V_{\text {eff }}$ is given by

$$
V_{\mathrm{eff}}=\frac{1}{\max \left(\epsilon(\boldsymbol{r})|\boldsymbol{e}(\boldsymbol{r})|^{2}\right)}
$$

where $e$ is the modal electric field traveling in the waveguide, $\epsilon(r)$ is the dielectric function that defines the periodic strcture and where $r$ is allowed to vary on the periodic cell. Figure 6 also reports the enhancement of $\operatorname{PF}$ (i.e. $\operatorname{PF}(a) / \operatorname{PF}\left(a_{0}\right)$, where $a$ is the increased periodicity compared to the nominal periodicity $a_{0}$, showing that it is mainly induced by the increase of group index.

A transition region between nominal and slow-light regimes can be realized easily by changing adiabatically the periodicity of the waveguide. Since the field profiles of the different regions are very similar, there is no need to modulate the waveguide width to spatially match the two modes, which has been shown to be a key aspect to obtain low insertion losses [40]. Yet, as discussed previously, the closeness of the photon energy to the photonic bandgap would make the system more sensitive to disorder and the fabrication more challenging, and will likely set the limitation of the slow-light operation.

The achievement of modest PF can benefit the field of quantum technologies based on color centres embedded in SiC. For example, the emission rate of the silicon vacancy $(\mathrm{SiV})$ center is limited by the non-radiative decay from the excited state to a metastable state [43]. Moreover, the collection efficiency in confocal microscopy setups is hampered by the high refractive index due to TIR. A moderate PF would increase the radiative rate to values sufficient to accomplish quantum non-demolition readout of the spin state.

\section{ELECTRO-OPTIC MODULATORS}

Active modulation of light travelling inside $\mathrm{SiC}$ can be performed with electro-optic modulators that exploit the high $\chi^{(2)}$ nonlinearity of the material. Assuming that the bottom surface of the structure is not accessible, the modulator could be realized by patterning two metallic pads to the sides of the suspending arms' region. In order to give an estimate of the performance of the device, we model the two pads as a parallel plate capacitor with $6 \mu \mathrm{m}$ spacing and centered on the $\mathrm{SiC}$ waveguide [44], so that the overlap between the driving and optical fields is equal to unity. Since the index of refraction of the material $n=\sqrt{1+\chi^{(1)}}$ is modified by an applied electric field $E$ according to

$$
n(E)=\sqrt{1+\chi^{(1)}+2 \chi^{(2)} E} \simeq n+\chi^{(2)} E,
$$

the standard voltage-length figure of merit for a $\pi$ phase shifter is given by

$$
L_{\pi} V_{\pi} \simeq \frac{\lambda l}{r n^{3}}
$$

where $l$ the distance between the capacitor plates and $r=$ $2 \chi^{(2)} / n^{4}$ is the electro-optic coefficient of the waveguide material. Assuming $n=2.6, \lambda_{0}=1550 \mathrm{~nm}, l=6 \mu \mathrm{m}, \chi^{(2)}=32.8$ $\mathrm{pm} / \mathrm{V}$, then $r=1.43 \mathrm{pm} / \mathrm{V}$ and $L_{\pi} V_{\pi} \simeq 36.9 \mathrm{~V} \cdot \mathrm{cm}$; this performance can be improved by a factor 2 by implementing an amplitude modulator based on a Mach-Zehnder interferometer driven by pads in the ground-signal-ground configuration, reducing $V_{\pi} L_{\pi}$ down to $18.4 \mathrm{~V} \cdot \mathrm{cm}$. This value is about one order of magnitude higher than the one of state of the art electro-optic modulators based on Lithium Niobate [45] $\left(r_{33} \simeq 30.8 \mathrm{pm} / \mathrm{V}\right.$ [27]) which use similar spacing between the pads [46]. As for 
other platforms, it is conceivable to improve the performance of electro-optic modulators using resonant structures like microring resonators [47]. We also confirmed that the pads induce negligible losses: a Lumerical simulation of the optical mode propagating alongside gold pads spaced by $6 \mu \mathrm{m}$ and placed outside the arms' region results in about $2 \mathrm{~dB} / \mathrm{m}$ losses.

\section{CONCLUSIONS}

In this work we proposed a scalable photonic platform based on $\mathrm{SiC}$ that allows coupling of electromagnetic radiation into and out of a SiC thin film and the manipulation of the electromagnetic field in the material. This platform, based on suspended subwavelength waveguides, is flexible enough for the realization of all the basic photonic components such as waveguides, bends, directional couplers, grating couplers and tapers. The proposed design requires a single etch step to access the substrate and to define the geometry of the devices, simplifying the fabrication process with respect to other previous $\mathrm{SiC}$ suspended platforms; despite this, the platform retains a powerful design flexibility, because the duty cycle of subwavelength sections can be different in different parts of the sample. As explained, an increase in the periodicity allows to reach a slow-light regime, which can be used to increase the linear and nonlinear interaction of light with $\mathrm{SiC}$ nonlinearities or color centers therein. For instance, this effect can be used to shorten the length of superconducting nanowires or electro-optical modulators integrated alongside the suspended waveguides.

Since $\mathrm{SiC}$ is very hard, compared to other sub-wavelength platforms realized in other materials such as $\mathrm{Si}$ and germanium, we believe that the lateral suspending structures can be very thin, hence allowing sub-wavelength regimes for shorter wavelengths than previously demonstrated. Reaching a periodicity shorter than $280 \mathrm{~nm}$ would permit the propagation of $1100 \mathrm{~nm}$ light, which in turn enables the interaction with NIR defects in SiC. Quantum optics applications would then become feasible. This, together with the increase of the $\beta$ factor given by slow-light could make this platform appealing for both $3 \mathrm{C}$ - and $4 \mathrm{H}-\mathrm{SiC}$. An even shorter periodicity of $200 \mathrm{~nm}$ would sustain the guided propagation of $785 \mathrm{~nm}$ radiation, the second harmonic of $1550 \mathrm{~nm}$; provided that a suitable way to obtain phase-matching between these two frequencies can be found, the strong $\chi^{(2)}$ nonlinearity of $\mathrm{SiC}$ would allow the efficient exploitation of second harmonic generation and stimulated/spontaneous parametric down-conversion.

Provided that the overall losses of the platform, given not only by the material but also by roughness and disorder, can be kept low enough, squeezing on an integrated, scalable platform would become a concrete and promising application.

\section{FUNDING}

Engineering and Physical Sciences Research Council (EPSRC) (EP/P003710/1)

\section{ACKNOWLEDGMENTS}

Useful discussions with Marco Liscidini are acknowledged.

\section{DISCLOSURES}

The authors declare no conflicts of interest.

\section{REFERENCES}

1. W. F. Koehl, B. B. Buckley, F. J. Heremans, G. Calusine, and D. D. Awschalom, "Room temperature coherent control of defect spin qubits in silicon carbide," Nature 479, 84 (2011).

2. A. L. Falk, B. B. Buckley, G. Calusine, W. F. Koehl, V. V. Dobrovitski, A. Politi, C. A. Zorman, P. X.-L. Feng, and D. D. Awschalom, "Polytype control of spin qubits in silicon carbide," Nat. Commun. 4, 1819 (2013).

3. S. Castelletto, B. Johnson, V. Ivády, N. Stavrias, T. Umeda, A. Gali, and T. Ohshima, "A silicon carbide room-temperature single-photon source," Nat. Mater. 13, 151 (2014).

4. M. Widmann, S.-Y. Lee, T. Rendler, N. T. Son, H. Fedder, S. Paik, L.-P. Yang, N. Zhao, S. Yang, I. Booker et al., "Coherent control of single spins in silicon carbide at room temperature," Nat. Mater. 14, 164 (2015).

5. D. J. Christle, A. L. Falk, P. Andrich, P. V. Klimov, J. U. Hassan, N. T. Son, E. Janzén, T. Ohshima, and D. D. Awschalom, "Isolated electron spins in silicon carbide with millisecond coherence times," Nat. Mater. 14, 160 (2015).

6. D. J. Christle, P. V. Klimov, F. Charles, K. Szász, V. Ivády, V. Jokubavicius, J. U. Hassan, M. Syväjärvi, W. F. Koehl, T. Ohshima et al., "Isolated spin qubits in $\mathrm{SiC}$ with a high-fidelity infrared spin-to-photon interface," Phys. Rev. X 7, 021046 (2017).

7. D. Simin, H. Kraus, A. Sperlich, T. Ohshima, G. Astakhov, and V. Dyakonov, "Locking of electron spin coherence above $20 \mathrm{~ms}$ in natural silicon carbide," Phys. Rev. B 95, 161201 (2017).

8. X. Tang, K. G. Irvine, D. Zhang, and M. G. Spencer, "Linear electrooptic effect in cubic silicon carbide," Appl. Phys. Lett. 59, 1938-1939 (1991).

9. I. Wu and G. Guo, "Second-harmonic generation and linear electrooptical coefficients of SiC polytypes and nanotubes," Phys. Rev. B 78, 035447 (2008).

10. O. Madelung, "Physics of group IV elements and III-V compounds," Landolt-Bornstein, Numer. Data Funct. Relationships Sci. Technol. New Ser. 17 (1982).

11. K. M. Jackson, J. Dunning, C. A. Zorman, M. Mehregany, and W. N. Sharpe, "Mechanical properties of epitaxial 3C silicon carbide thin films," J. microelectromechanical systems 14, 664-672 (2005).

12. I. Chatzopoulos, F. Martini, R. Cernansky, and A. Politi, "High-Q/V photonic crystal cavities and QED analysis in 3C-SiC," ACS Photonics 6, 1826-1831 (2019).

13. J. D. Caldwell, O. J. Glembocki, Y. Francescato, N. Sharac, V. Giannini, F. J. Bezares, J. P. Long, J. C. Owrutsky, I. Vurgaftman, J. G. Tischler et al., "Low-loss, extreme subdiffraction photon confinement via silicon carbide localized surface phonon polariton resonators," Nano letters 13, 3690-3697 (2013).

14. T. Fan, H. Moradinejad, X. Wu, A. A. Eftekhar, and A. Adibi, "High-Q integrated photonic microresonators on 3C-SiC-on-insulator (SiCOI) platform," Opt. Express 26, 25814-25826 (2018).

15. L. Di Cioccio, Y. Le Tiec, F. Letertre, C. Jaussaud, and M. Bruel, "Silicon carbide on insulator formation using the Smart Cut process," Electron. Lett. 32, 1144-1145 (1996).

16. B.-S. Song, T. Asano, S. Jeon, H. Kim, C. Chen, D. D. Kang, and S. Noda, "Ultrahigh-Q photonic crystal nanocavities based on $4 \mathrm{H}$ silicon carbide," Optica. 6, 991-995 (ts).

17. D. M. Lukin, C. Dory, M. A. Guidry, K. Y. Yang, S. D. Mishra, R. Trivedi, M. Radulaski, S. Sun, D. Vercruysse, G. H. Ahn et al., "4H-siliconcarbide-on-insulator for integrated quantum and nonlinear photonics," Nat. Photonics (2019).

18. D. O. Bracher and E. L. Hu, "Fabrication of high-Q nanobeam photonic crystals in epitaxially grown 4H-SiC," Nano Lett. 15, 6202-6207 (2015).

19. G. Calusine, A. Politi, and D. D. Awschalom, "Silicon carbide photonic crystal cavities with integrated color centers," Appl. Phys. Lett. 105, 011123 (2014).

20. F. Martini and A. Politi, "Four wave mixing in $3 \mathrm{C}$ SiC ring resonators," Appl. Phys. Lett. 112, 251110 (2018).

21. J. S. Penadés, C. Alonso-Ramos, A. Khokhar, M. Nedeljkovic, L. Boodhoo, A. Ortega-Moñux, I. Molina-Fernández, P. Cheben, and G. Mashanovich, "Suspended soi waveguide with sub-wavelength grat- 
ing cladding for mid-infrared," Opt. Lett. 39, 5661-5664 (2014).

22. J. S. Penades, A. O.-M. nux, M. Nedeljkovic, J. G. Wangüemert-Pérez, R. Halir, A. Z. Khokhar, C. Alonso-Ramos, Z. Qu, I. Molina-Fernández, P. Cheben, and G. Z. Mashanovich, "Suspended silicon mid-infrared waveguide devices with subwavelength grating metamaterial cladding," Opt. Express 24, 22908-22916 (2016).

23. J. S. Penadés, A. Sánchez-Postigo, M. Nedeljkovic, A. Ortega-Moñux, J. Wangüemert-Pérez, Y. Xu, R. Halir, Z. Qu, A. Khokhar, A. Osman et al., "Suspended silicon waveguides for long-wave infrared wavelengths," Opt. Lett. 43, 795-798 (2018).

24. W. Zhou, Z. Cheng, X. Wu, X. Sun, and H. K. Tsang, "Fully suspended slot waveguide platform," J. Appl. Phys. 123, 063103 (2018).

25. P. Cheben, R. Halir, J. H. Schmid, H. A. Atwater, and D. R. Smith, "Subwavelength integrated photonics," Nature. 560, 565 (2018).

26. J. D. Joannopoulos, R. D. Meade, and J. N. Winn, Photonic Crystals: Molding The Flow of Light (Princeton, 1995).

27. A. Yariv and P. Yeh, "Photonics: optical electronics in modern communications (the oxford series in electrical and computer engineering)," Oxf. Univ. Press. Inc 231, 232 (2006).

28. S. G. Johnson and J. D. Joannopoulos, "Block-iterative frequencydomain methods for Maxwell's equations in a planewave basis," Opt. Express 8, 173-190 (2001).

29. F. Payne and J. Lacey, "A theoretical analysis of scattering loss from planar optical waveguides," Opt. Quantum Electron. 26, 977-986 (1994).

30. F. Grillot, L. Vivien, S. Laval, D. Pascal, and E. Cassan, "Size influence on the propagation loss induced by sidewall roughness in ultrasmall SOI waveguides," IEEE Photonics Technol. Lett. 16, 1661-1663 (2004).

31. A. Ortega-Moñux, J. Čtyrokỳ, P. Cheben, J. H. Schmid, S. Wang, Í. Molina-Fernández, and R. Halir, "Disorder effects in subwavelength grating metamaterial waveguides," Opt. Express 25, 12222-12236 (2017).

32. M. A. Foster, K. D. Moll, and A. L. Gaeta, "Optimal waveguide dimensions for nonlinear interactions," Opt. Express 12, 2880-2887 (2004).

33. T. Sato, S. Makino, Y. Ishizaka, T. Fujisawa, and K. Saitoh, "A rigorous definition of nonlinear parameter $\gamma$ and effective area $A_{\text {eff }}$ for photonic crystal optical waveguides," JOSA B 32, 1245-1251 (2015).

34. D. Tan, K. Ikeda, P. Sun, and Y. Fainman, "Group velocity dispersion and self phase modulation in silicon nitride waveguides," Appl. Phys. Lett. 96, 061101 (2010).

35. R. Halir, P. Cheben, S. Janz, D.-X. Xu, Í. Molina-Fernández, and J. G. Wangüemert-Pérez, "Waveguide grating coupler with subwavelength microstructures," Opt. Lett. 34, 1408-1410 (2009).

36. Z. Cheng, X. Chen, C. Y. Wong, K. Xu, and H. K. Tsang, "Broadband focusing grating couplers for suspended-membrane waveguides," Opt. Lett. 37, 5181-5183 (2012).

37. R. Halir, P. Cheben, J. Schmid, R. Ma, D. Bedard, S. Janz, D.-X. Xu, A. Densmore, J. Lapointe, and I. Molina-Fernández, "Continuously apodized fiber-to-chip surface grating coupler with refractive index engineered subwavelength structure," Opt. Lett. 35, 3243-3245 (2010).

38. A. Sánchez-Postigo, J. G. Wangüemert-Pérez, J. S. Penadés, A. Ortega-Moñux, M. Nedeljkovic, R. Halir, F. E. M. Mimun, Y. X. Cheng, Z. Qu, A. Z. Khokhar et al., "Mid-infrared suspended waveguide platform and building blocks," IET Optoelectronics 13, 55-61 (2018).

39. A. Sánchez-Postigo, A. Ortega-Moñux, D. Pereira-Martín, Í. MolinaFernández, R. Halir, P. Cheben, J. S. Penadés, M. Nedeljkovic, G. Mashanovich, and J. Wangüemert-Pérez, "Design of a suspended germanium micro-antenna for efficient fiber-chip coupling in the longwavelength mid-infrared range," Opt. express 27, 22302-22315 (2019).

40. T. F. Krauss, "Slow light in photonic crystal waveguides," J. Phys. D: Appl. Phys. 40, 2666 (2007).

41. V. M. Rao and S. Hughes, "Single quantum-dot Purcell factor and $\beta$ factor in a photonic crystal waveguide," Phys. Rev. B 75, 205437 (2007).

42. M. Arcari, I. Söllner, A. Javadi, S. L. Hansen, S. Mahmoodian, J. Liu, H. Thyrrestrup, E. H. Lee, J. D. Song, S. Stobbe et al., "Near-unity coupling efficiency of a quantum emitter to a photonic crystal waveguide," Phys. Rev. Lett. 113, 093603 (2014).

43. R. Nagy, M. Niethammer, M. Widmann, Y.-C. Chen, P. Udvarhelyi,
C. Bonato, J. U. Hassan, R. Karhu, I. G. Ivanov, N. T. Son et al., "Highfidelity spin and optical control of single silicon-vacancy centres in silicon carbide," Nat. communications 10 (2019).

44. R. C. Alferness, "Waveguide electrooptic modulators," IEEE Transactions on Microw. Theory Tech. 30, 1121-1137 (1982).

45. C. Wang, M. Zhang, B. Stern, M. Lipson, and M. Lončar, "Nanophotonic lithium niobate electro-optic modulators," Opt. express 26, 1547-1555 (2018).

46. D. Janner, D. Tulli, M. García-Granda, M. Belmonte, and V. Pruneri, "Micro-structured integrated electro-optic $\mathrm{LiNbO}_{3}$ modulators," Laser \& Photonics Rev. 3, 301-313 (2009).

47. Q. Xu, B. Schmidt, S. Pradhan, and M. Lipson, "Micrometre-scale silicon electro-optic modulator," Nature. 435, 325 (2005). 